Original Article

\title{
Long Term Results and Predictors of Left Ventricular Function Recovery after Aortic Valve Replacement for Chronic Aortic Regurgitation
}

\author{
Hiroyuki Saisho, MD, ${ }^{1}$ Koichi Arinaga, MD, PhD,${ }^{1}$ Satoshi Kikusaki, MD, ${ }^{1}$ \\ Yuichiro Hirata, MD, ${ }^{1}$ Kumiko Wada, MD, ${ }^{1}$ Tatsuyuki Kakuma, PhD, ${ }^{2}$ and \\ Hiroyuki Tanaka, MD, $\mathrm{PhD},{ }^{1}$
}

\begin{abstract}
Objectives: In most patients with aortic regurgitation (AR), aortic valve replacement (AVR) improves left ventricular ( $L V)$ function, but some patients will not have favorable remodeling. Our objectives were to review long term clinical results of AVR for AR and to examine what factors affect the normalization of $L V$ function after AVR for chronic AR. Methods: Between 1989 and 2010, 177 patients underwent isolated AVR for chronic pure AR. The patients were divided into 2 groups based on indexed end-systolic $L V$ diameter (iESD): Group L (iESD) $\geqq 25 \mathrm{~mm} / \mathrm{m}^{2}$ ) (130 patients) and Group $S$ (iESD $<25 \mathrm{~mm} / \mathrm{m}^{2}$ ) (47 patients).

Results: There was no significant difference between groups in late mortality, freedom from cardiac-related death and rehospitalization for heart failure at late follow up after operation. At postoperative follow-up, $16 \%$ of patients had not recovered normal LV systolic function. By means of multivariate analysis, iESD and cardiac index (CI) were independent predictors of recovery of $L V$ function and $\mathrm{iESD}>26.7 \mathrm{~mm} / \mathrm{m}^{2}$ and $\mathrm{CI}<2.71 \mathrm{l} / \mathrm{min} / \mathrm{m}^{2}$ were the best cut-off values.

Conclusions: Early and late surgical results of AVR for chronic AR were good, but for the preservation of postoperative normal $\mathrm{LV}$ function, AVR for AR patients should be performed before iESD reaches $26.7 \mathrm{~mm} / \mathrm{m}^{2}$.
\end{abstract}

Keywords: Isolated aortic valve replacement, Chronic aortic regurgitation, body surface area (BSA), Left ventricular function, timing of operation

\section{Introduction}

Chronic aortic regurgitation (AR) involves both volume and pressure overload at the left ventricle (LV) and the

${ }^{1}$ Department of Surgery, Kurume University School of Medicine, Kurume, Fukuoka, Japan

${ }^{2}$ Biostatistics Center, Kurume University, Kurume, Fukuoka, Japan

Received: October, 27, 2014; Accepted: December 9, 2014

Corresponding author: Hiroyuki Tanaka, Department of Surgery,

Kurume University School of Medicine, 67 Asahi-machi, Kurume,

Fukuoka 830-0011, Japan

Email: htanaka@med.kurume-u.ac.jp

(C)2014 The Editorial Committee of Annals of Thoracic and

Cardiovascular Surgery. All rights reserved. goals of treatment are to prevent death, diminish symptoms, and prevent the development of heart failure. ${ }^{1)}$

Aortic valve replacement (AVR) is an established treatment for AR and the long term results of AVR for chronic AR are satisfactory. However, in some cases postoperative LV dilatation and function are inadequate. Indeed, Henry, et al. showed that cases with end-systolic LV diameter (ESD) $\geqq 55 \mathrm{~mm}$ and $\%$ fractional shortening $<25 \%$ had poor prognosis and more frequent post-operative complications. ${ }^{2)}$

LV systolic function and LV dimensions have been regarded as important parameters when considering appropriate timing of AVR for patients with AR. ${ }^{3)}$ However, it is unclear when the operation should be undertaken in such patients. 
Recent studies have suggested the use of indexed LV dimensions in chronic AR to optimize surgical indications because decisions based on raw data might be inappropriate for patients with extreme body surface area (BSA) such as Japanese. ${ }^{47)}$ In the 2012 The European Society of Cardiology/The European Association for Cardio-Thoracic Surgery (ESC/EACTS) guidelines, indexed ESD (iESD) was newly added as a parameter for class IIa indication of AVR for AR: surgery for severe AR should be considered in asymptomatic patients with ejection fraction (EF) $>50 \%$ ) with severe LV dilatation (end diastolic diameter (EDD) $>70 \mathrm{~mm}$ or end systolic diameter $(\mathrm{ESD})>50 \mathrm{~mm}$ or indexed ESD (iESD) $>25 \mathrm{~mm} / \mathrm{m}^{2}$ with small body size). In a recent revision of the The American Heart Association/ The American College of Cardiology (AHA/ACC) guidelines, iESD was newly included as a decision-making criterion for operation regardless of body size. ${ }^{8)}$ However, there are few studies examining predictive factors for prognosis after AVR for AR in Japanese patients.

In this study, we retrospectively reviewed 177 patients who underwent AVR for AR at our hospital for 22 years and evaluated the early and long term clinical results of AVR for AR and examined several predictors of recovery of LV function after the operation.

\section{Patients and Methods}

The Ethics Committee of Kurume University School of Medicine and the Institutional Review Board approved this study. Individual patient consent was waived because the study was retrospective.

\section{Patients}

From 1989 to 2010, 177 patients underwent primary isolated AVR for chronic pure AR at Kurume University Hospital. Indications of AVR were symptomatic severe AR and asymptomatic severe AR with LV systolic dysfunction $(\mathrm{EF} \leqq 50 \%$ ) or dilatation ( $\mathrm{EF}>50 \%$ with $\mathrm{EDD}$ $>70 \mathrm{~mm}$ and ESD $>50 \mathrm{~mm}$ ). To select only patients with chronic pure severe AR, patients were excluded who had mild or greater aortic stenosis or local asynergy of wall motion on preoperative transthoracic echocardiography (TTE). Patients were also excluded who had previous cardiac surgery, concomitant coronary artery bypass graft, other heart valve surgery or aortic surgery. Moreover, patients with acute AR, including aortic dissections or infective endocarditis, were also excluded.

Subjects were 122 men and 55 women with a mean age of $58.4 \pm 15.4$ years (range, 21 to 84 years). The mean BSA
Was $1.57 \pm 0.17 \mathrm{~m}^{2}$ (range, 1.18 to $2.00 \mathrm{~m}^{2}$ ). The etiologies of AR were degeneration in 97 patients (55\%), congenital anomaly in 39 patients (22\%; bicuspid valve 32 , quadricuspid valve 7), rheumatic fever in 20 patients (11\%), annular dilatation in 14 patients $(8 \%)$ and aortitis in 7 patients $(4 \%)$.

Preoperative variables are shown in Table 1. We divided these patients into 2 groups based on iESD (Group L: iESD $>25 \mathrm{~mm} / \mathrm{m}^{2}$, Group S: iESD $\leqq 25 \mathrm{~mm} / \mathrm{m}^{2}$ ) and 130 patients $\left(73.4 \%\right.$ ) had LV dilatation of $\mathrm{iESD}>25 \mathrm{~mm} / \mathrm{m}^{2}$.

\section{Data Collection and Follow-Up}

Preoperative and perioperative data were collected from medical records and follow-up data were obtained from our outpatient clinic records or by correspondence with referring physicians. Hospital survivors were followed up annually at our outpatient clinic. Patients were contacted directly by telephone or through the referring physician, and additional data were collected from clinical records. Deaths and complications were properly defined according to published guidelines of The Society of Thoracic Surgeons.9) EF and LV dimensions were measured and the normalization of LV systolic function was defined as $\mathrm{EF} \geqq 55 \%$ according to recommendations by the American Society of Echocardiography. ${ }^{10)}$

\section{Statistical Methods}

Continuous variables were expressed as mean \pm standard deviation. Simple comparisons were performed using a standard $\chi^{2}$ test or a non-paired $t$ test. The paired t-test was used to compare 2 groups, while analysis of variance (ANOVA) was used to compare more than 2 groups. Survivals and freedom from late complications were estimated using the Kaplan-Meier method. Survival curves were compared using a log-rank test. The risk factors for recovery of LV systolic function were analyzed by both univariate and multivariate analysis with the Cox proportional hazards model. For continuous variables, diagnostic cutoff values with the most favorable sensitivities and specificities were identified by receiver operating characteristics (ROC) curve analysis.

\section{Results}

\section{Clinical Outcome}

There were two hospital deaths (1.1\%), caused by low cardiac output syndrome and pneumonia respectively and both were Group L.

Among the 175 operative survivors, 168 patients (96.0\%) were followed up. The mean follow-up period 
Table 1 Comparison of preoperative patients' characteristics

\begin{tabular}{lcccc}
\hline Characteristics & All patients & Group L & Group S & P value \\
\hline Number of patients & 177 & 130 & 47 & \\
Age (years) & $58.4 \pm 15.4$ & $58.7 \pm 15.4$ & $57.7 \pm 15.6$ & 0.71 \\
& $(21 \sim 84)$ & $(26 \sim 84)$ & $(21 \sim 84)$ & \\
Male/Female & $122 / 55$ & $90 / 40$ & $32 / 15$ & 0.83 \\
BSA $\left(\mathrm{m}^{2}\right)$ & $1.57 \pm 0.17$ & $1.56 \pm 0.16$ & $1.60 \pm 0.17$ & 0.11 \\
NYHA III or IV & $37(20.9 \%)$ & $26(20.0 \%)$ & $11(23.4 \%)$ & 0.09 \\
EDD $(\mathrm{mm})$ & $63.6 \pm 8.5$ & $66.0 \pm 8.1$ & $57.1 \pm 5.8$ & $<0.01$ \\
& $(45 \sim 88)$ & $(47 \sim 88)$ & $(45 \sim 67)$ & \\
iEDD $\left(\mathrm{mm} / \mathrm{m}^{2}\right)$ & $40.9 \pm 6.0$ & $42.9 \pm 5.5$ & $35.4 \pm 3.1$ & $<0.01$ \\
& $(29 \sim 62)$ & $(35 \sim 62)$ & $(29 \sim 42)$ & \\
ESD $(\mathrm{mm})$ & $43.7 \pm 8.6$ & $46.7 \pm 7.9$ & $35.6 \pm 4.4$ & $<0.01$ \\
& $(28 \sim 68)$ & $(32 \sim 68)$ & $(28 \sim 45)$ & \\
iESD $\left(\mathrm{mm} / \mathrm{m}^{2}\right)$ & $28.2 \pm 6.0$ & $30.4 \pm 5.5$ & $22.1 \pm 1.8$ & - \\
& $(17 \sim 49)$ & $(25 \sim 49)$ & $(17 \sim 24)$ & \\
EF $(\%)$ & $58.0 \pm 10.3$ & $54.8 \pm 9.7$ & $66.6 \pm 6.0$ & $<0.01$ \\
& $(31 \sim 74)$ & $(31 \sim 72)$ & $(57 \sim 79)$ & \\
\hline
\end{tabular}

Group L: iESD > 25 mm/m², Group S: iESD $\leqq 25 \mathrm{~mm} / \mathrm{m}^{2}$; P value: Group L vs Group S. BSA: body surface area; NYHA: New York Heart Association; EDD: end diastolic diameter; iEDD: indexed end diastolic diameter; ESD: end systolic diameter; iESD: indexed end systolic diameter; EF: ejection fraction

was $11.8 \pm 6.5$ years (range, 2 months to 24.8 years) for a total of 2081.3 patient-years. During the follow-up period, 38 patients $(21.5 \%)$ died of various causes, including 4 cardiac-related deaths $(2.3 \%)$ (heart failure in three patients, arrhythmia in one patient). Of all cardiac-related deaths, three were in Group L and one was in Group S.

The actuarial survival, including early death at 10 and 20 years was $89.9 \%$ and $55.2 \%$ in Group L, and $82.7 \%$ and $71.6 \%$ in Group $\mathrm{S}(\mathrm{P}=0.72)$, respectively. Differences in the actuarial survival were not significant (Fig. 1A).

The actuarial freedom from cardiac-related death at 10 and 20 years was $99.2 \%$ and $97.5 \%$ in Group L, and $95.0 \%$ and $79.1 \%$ in Group $\mathrm{S}(\mathrm{P}=0.25)$, respectively. Differences in the actuarial freedom from cardiacrelated death were not significant (Fig. 1B).

Eight patients were admitted to our hospital due to heart failure for an incidence of $0.4 \%$ per patient-year. Of them, five patients were in Group L, and three patients were Group S. The details of rehospitalization due to heart failure are presented in Table $\mathbf{2}$. The actuarial freedom from rehospitalization due to heart failure at 10 and 20 years was $98.0 \%$ and $92.4 \%$ in Group L, and $97.2 \%$ and $71.8 \%$ in Group S $(\mathrm{P}=0.31)$, respectively. Differences in the freedom from rehospitalization due to heart failure were not significant (Fig. 1C).

\section{Time course of Echocardiographic Data}

Echocardiography was performed within 1 week before the operation, at the immediate postoperative period (7 days after the operation), at early follow up (1 year after the operation) and at late follow-up period (mean 6.7 years (range, 3 years to 10 years)). Figure 2 shows the variables before and after the operation in the two groups. The mean iEDD and iESD in both groups just after the operation were significantly improved from preoperative values (iEDD: $42.9 \pm 5.4$ to $34.2 \pm 4.7 \mathrm{~mm} / \mathrm{m}^{2}$, iESD: $30.4 \pm 5.5$ to $24.9 \pm 4.9 \mathrm{~mm} / \mathrm{m}^{2}$ in Group L, and iEDD: $35.4 \pm 3.1$ to $31.1 \pm 3.5 \mathrm{~mm} / \mathrm{m}^{2}$, iESD: $22.1 \pm 1.8$ to $20.8 \pm 3.6 \mathrm{~mm} / \mathrm{m}^{2}$ in Group S). The improvement in these variables was maintained in both groups at early postoperative follow-up and at long term period. The mean iEDD and iESD in Group L were significantly larger at the immediate postoperative period, but after that, there were no significant differences between the two groups.

The mean preoperative EF was $54.8 \pm 9.7 \%$ in Group L and $66.6 \pm 6.0 \%$ in Group $\mathrm{S}(\mathrm{P}<0.05)$. There was a temporal decrease in both groups immediately after the operation, but it improved and maintained normal values during follow up, and no significant difference was recognized between the two groups.

\section{Predictors of the normalization of LV systolic function}

Postoperative follow-up echocardiograms were obtained in 144 of the 177 patients and 23 of these patients $(16.0 \%)$ were unable to recover to the normal value of EF. Several factors affecting recovery of postoperative LV 
(A)
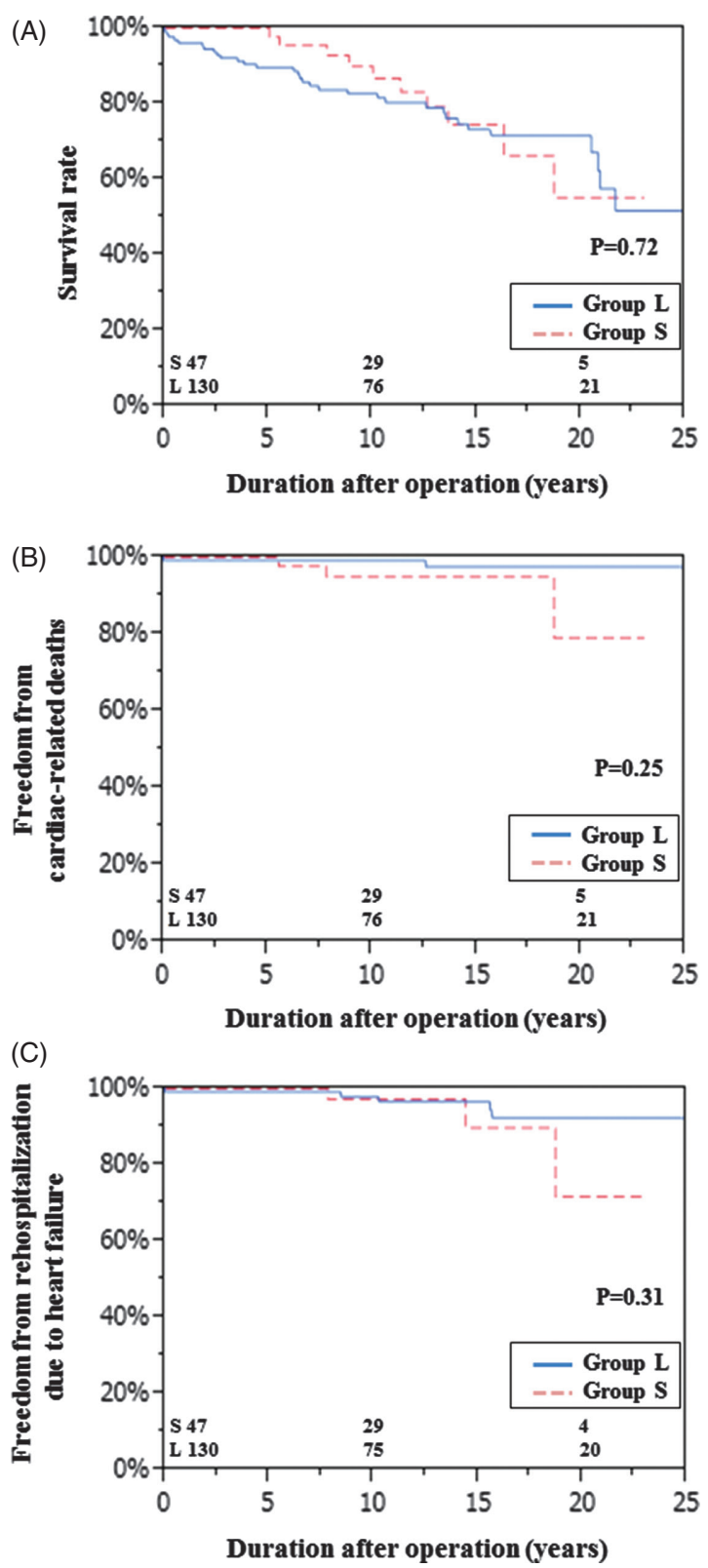

Fig. 1 (A) Actuarial survival rate. (B) Freedom from cardiac-related deaths. (C) Freedom from rehospitalization due to heart failure. Group L: iESD $>25 \mathrm{~mm} / \mathrm{m}^{2}$, Group $\mathrm{S}: \mathrm{iESD} \leqq 25 \mathrm{~mm} / \mathrm{m}^{2}$.

systolic function were identified by univariate and multivariate analyses (Table 3).

By means of univariate analysis, we determined that ESD, iESD, EF, cardiac index (CI), history of atrial fibrillation (AF) and smoking were associated with postoperative normalization of EF. EDD and iEDD were not predictive of normal $\mathrm{EF}$ in the postoperative period. By means of multivariate analysis, $\mathrm{EESD}$ and $\mathrm{CI}$ were found to be significant factors and patients with lower $\mathrm{EF}$, history of $\mathrm{AF}$ and habit of smoking had poorer recovery of LV systolic function.

Receiver operating characteristic curve analyses showed that the best cut-off values for predicting of EF recovery after surgery were $\mathrm{iESD}$ of $26.7 \mathrm{~mm} / \mathrm{m}^{2}$, EF of $50.2 \%$, and CI of $2.71 \mathrm{l} / \mathrm{min} / \mathrm{m}^{2}$.

To score the risk of poor recovery of LV systolic function with the above factor, we assigned two points to a larger iESD $\left(\geqq 26.7 \mathrm{~mm} / \mathrm{m}^{2}\right)$, lower EF $(<50.2 \%)$, history of $\mathrm{AF}$ and habit of smoking and three points to lower CI $\left(<2.71 \mathrm{l} / \mathrm{min} / \mathrm{m}^{2}\right)$. As a result, we found that patients with a score of five or more had poor recovery of LV systolic function after AVR, with a sensitivity and specificity of $82.6 \%$ and $64.5 \%$, respectively.

\section{Discussion}

In the new 2012 ESC/EACTS guidelines, $\mathrm{iESD}$ was used as a parameter for decision making for AVR for chronic AR, that is, asymptomatic patients with severe $\mathrm{AR}$ and severe LV dilatation (iESD $>25 \mathrm{~mm} / \mathrm{m}^{2}$ ) were newly added as a class IIa indication for AVR. This revision was based on a report that the use of $\mathrm{IESD}$ improves the prediction of unfavorable outcome after AVR in patients with low BSA and that iESD $>25 \mathrm{~mm} / \mathrm{m}^{2}$ should be used as a cut-off point for surgery rather than ESD $>50 \mathrm{~mm}$ in patients with low BSA. ${ }^{4)}$ Indeed, Daimon et al. showed that BSA of Japanese is very low and LV dimensions are small compared with the reference values in the guidelines from the American Society of Echocardiography. ${ }^{11)}$ In this study, the mean BSA was $1.57 \pm$ $0.17 \mathrm{~m}^{2}$, which demonstrates that our patients were smaller than those in other reports, especially those from Western countries. ${ }^{611,12)}$ This difference in physique between Japanese and Western populations will have a great effect on how the grade of LV dilatation influences the results of AVR.

In this study, we found that $\mathrm{iESD}$ of $26.7 \mathrm{~mm} / \mathrm{m}^{2}$ was the best cut-off values for predicting EF recovery after surgery, and this result supports the new guideline from the viewpoint of the LV reverse remodeling effect. However, there was no significant factor in late survival, cardiacrelated death and freedom from rehospitalization due to heart failure despite the use of indexed LV dimensions.

The present results revealed that patients who had isolated AVR for chronic AR had good clinical results regardless of the severity of LV dilatation and hospital mortality at our hospital $(1.1 \%)$ was lower than other previous studies $(1.7 \%$ to $14 \%),{ }^{4,5,6,13,14)}$ and late survival was 
Table 2 Summary of eight rehospitalized patients

\begin{tabular}{|c|c|c|c|c|c|}
\hline Patient & $\begin{array}{l}\text { Pre-op iEDD/iESD } \\
\qquad\left(\mathrm{mm} / \mathrm{m}^{2}\right)\end{array}$ & Pre-op EF (\%) & Cause of rehospitalization & Outcome & $\begin{array}{c}\text { Duration from } \\
\text { surgery (mo) }\end{array}$ \\
\hline $64 \mathrm{M}$ & $35 / 19$ & 75 & $\mathrm{CHF}$ following thrombosed valve & death & 66 \\
\hline $67 \mathrm{M}$ & $57 / 40$ & 51 & CHF with exacerbation of MR & death & 93 \\
\hline $47 \mathrm{M}$ & $56 / 44$ & 43 & $\begin{array}{l}\text { CHF due to difficulty with } \\
\text { volume control for HD }\end{array}$ & death & 101 \\
\hline $66 \mathrm{~F}$ & $37 / 24$ & 64 & $\begin{array}{c}\text { CHF with new occurrenceof } \mathrm{AF} \text { and } \\
\text { exacerbation of MR }\end{array}$ & death & 234 \\
\hline $69 \mathrm{M}$ & $37 / 29$ & 42 & CHF with exacerbation of MR & alive & 128 \\
\hline $72 \mathrm{M}$ & $35 / 24$ & 60 & unknown & alive & 174 \\
\hline $51 \mathrm{M}$ & $40 / 30$ & 48 & CHF with exacerbation of MR & alive & 186 \\
\hline $40 \mathrm{M}$ & $45 / 40$ & 27 & CHF with CRT for trifasoicular block & alive & 186 \\
\hline
\end{tabular}

iEDD: indexed end diastolic diameter; iESD: indexed end systolic diameter; EF: ejection fraction; CHF: congestive heart failure; MR: mitral regurgitation; HD: hemodialysis; AF: atrial fibrillation; CRT: cardiac resynchronization therapy; mo: month

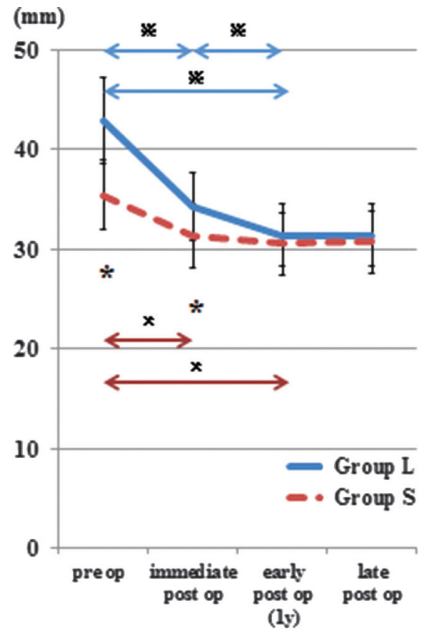

(A)

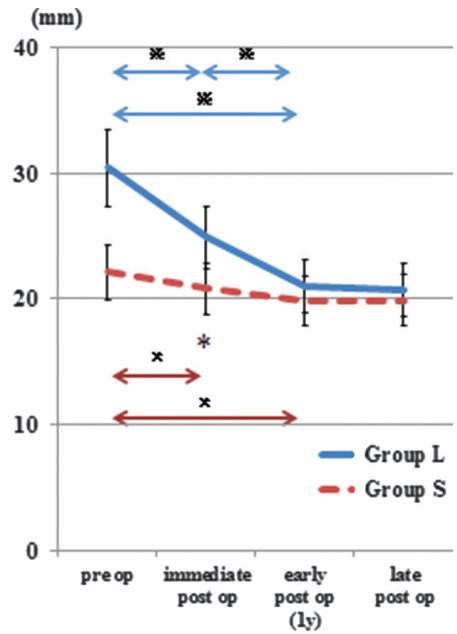

(B)

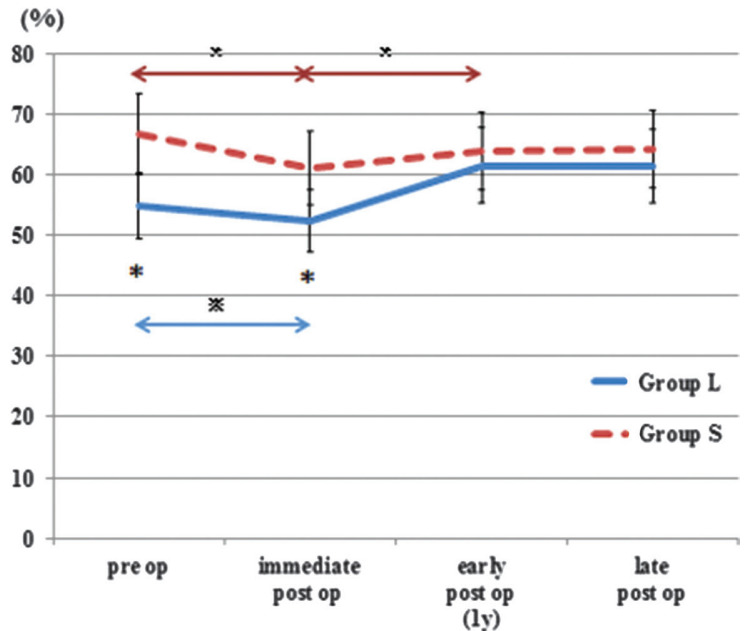

(C)

Fig. 2 (A) Preoperative, immediate postoperative, early postoperative and late postoperative iEDD. (B) Preoperative, immediate postoperative, early postoperative and late postoperative iESD. (C) Preoperative, immediate postoperative, early postoperative and late postoperative EF. iEDD: indexed end diastolic diameter; iESD: indexed end systolic diameter; EF: ejection fraction; op: operation; 1y: 1 year; Group L: iESD $>25 \mathrm{~mm} / \mathrm{m}^{2}$, Group S: iESD $\leqq 25 \mathrm{~mm} / \mathrm{m}^{2}$. *: Significant difference $(\mathrm{p}<0.05)$ between 2 groups. $※: \mathrm{p}<0.05$ between 2 periods in Group L. $\times$ : $\mathrm{p}<0.05$ between 2 periods in Group S.

good. These good results are thought to be due to improved operative techniques, intensive care and medical therapy, as well as low incidence of cardiac-related complications.

The timing of operation for asymptomatic severe chronic AR remains controversial. Preoperative ESD has been reported as a poor prognostic factor in patients with severe AR after AVR. Henry, et al. showed that cases with $\mathrm{ESD} \geqq 55 \mathrm{~mm}$ and $\%$ fractional shortening $<25 \%$ had a poor prognosis and more frequent post-operative complications. ${ }^{3)}$ Some clinicians looked negatively at the operation because they believed myocardial changes in these patients to be at an irreversible stage. ${ }^{15)}$ The Euro Heart Survey revealed that only $22 \%$ of chronic AR patients with left ventricular ejection fraction (LVEF) of from 30 to $50 \%$ received the operation and only $3 \%$ of those with LVEF <30\% did. ${ }^{16)}$ This situation may be because of the clear relationship between lower preoperative LVEF and worse postoperative survival. Therefore, $55 \mathrm{~mm}$ of ESD has been widely accepted as a cut off value indicating poor prognosis and there is still a major reluctance to offer AVR to these patients in the current era.

Even though many of them develop irreversible changes, some cases manifest a meaningful recovery of LV function after AVR. ${ }^{14,15,17,18)}$ Some studies showed there was no significance difference in survival rate between cases with severe LV dysfunction and those 
Table 3 Multivariable Cox proportional hazards regression model of recovery of $\mathrm{LV}$ systolic function

\begin{tabular}{lcc}
\hline Variable & $\begin{array}{c}\text { Odds ratio } \\
(95 \% \text { Confidence Interval })\end{array}$ & P value \\
\hline iESD & $3.72[1.05-13.2]$ & 0.04 \\
EF & $3.14[0.92-10.71]$ & 0.06 \\
CI & $4.76[1.38-16.39]$ & 0.01 \\
AF & $5.22[0.99-27.46]$ & 0.05 \\
smoking & $2.82[0.93-8.53]$ & 0.06 \\
\hline
\end{tabular}

LV: left ventricle; iESD: :indexed end systolic diameter; EF: ejection fraction; $\mathrm{CI}$ : cardiac index; AF: atrial fibrillation

with normal LV function after AVR. ${ }^{19,20)}$ Our study had 18 patients $(10.2 \%)$ with $\mathrm{ESD} \geqq 55 \mathrm{~mm}$ and there was no operative mortality and no significant difference in postoperative course at late follow up compared with patients with ESD $<55 \mathrm{~mm}$.

The current ACC/AHA guidelines do not recommend AVR for patients with LVEF $>50 \%$, EDD $<70 \mathrm{~mm}$, and ESD $<50 \mathrm{~mm}$ in the absence of concomitant cardiac or aortic surgery, ${ }^{21)}$ and few studies have evaluated the impact of AVR on survival in the asymptomatic phase of the disease. Turk et al. investigated whether AVR would yield a survival benefit in asymptomatic severe AR patients with those parameter values and showed that AVR was associated with significant survival benefit. ${ }^{22)}$ Considering the declining operative mortality of AVR and the improvement of perioperative management, these results possibly support a strategy of earlier surgery for $\mathrm{AR}$ and despite serving as a class III indication, AVR could be indicated in severe asymptomatic AR patients with normal LVEF and lesser LV dilatation.

AR is the combined condition of volume overload and pressure overload and LVEF and ESD have been focused on as measures of LV pump performance and EDD as a measure of the severity of the volume overload in asymptomatic patients with AR. After the removal of this condition with surgical repair, enlarged LV dimensions, mainly EDD, are usually reduced within a few weeks after the operation, because the effect of preload decrease appears to be dominant at this time. Therefore, LVEF decreases temporarily. ${ }^{22-24)}$ In our study, both groups had great reduction in iEDD and iESD, but the decrease in iESD in Group S was smaller than in Group L. That's why the grade of LVEF decline was greater in Group L but Group S had significantly better LVEF than Group L. After discharge, Group L had additional improvement in LV dimensions, and LV function reached a plateau at the early period after the operation. This chronological change could result from the reduced afterload with gradual improvement in LV function after surgery or it might show an actual improvement in contractile function and would indicate that it takes about one year to make sure of the improvement of LV function with AVR. That is why we choose this period as the appropriate timing for judging the effect of the operation.

The other predictors we identified, CI, history of AF and habit of smoking should also be considered in assessing the postoperative prognosis of these patients.

In the presence of severe AR, stroke volume (SV) depends on the regurgitant volume and on the pumping capacity of LV. Some studies have clearly suggested a link between SV and the presence and extent of viable myocardium in patients with heart failure from systolic dysfunction. ${ }^{25,26)} \mathrm{CI}$ is associated with $\mathrm{SV}$ in this condition, and the preservation of elevated CI with decreased systolic function could suggest that there is enough viable myocardium and the operation can lead this heart to eventual favorable remodeling.

There are few studies about the relationship between $\mathrm{AF}$, habit of smoking and recovery of LV function after surgery. Persistent AF is reported to be independently associated with increasing diffuse fibrosis of LV and AF itself may play an independent role in adverse cardiac remodeling. ${ }^{27)}$ Smoking that includes more than 4000 chemical substances, promotes cardiomyocyte apoptosis by inducing oxidative stress and is suggested to be related to LV dysfunction. ${ }^{28,29)}$ Further studies are necessary to evaluate how these conditions affect the postoperative clinical course.

To our knowledge, this is the first study to evaluate the effect of AVR on LV reverse remodeling from the point of preoperative factors including hemodynamics indices such as iESD and CI and to score the risk in Japanese patients. These days, the improvement in operative results is shifting the timing of AVR for AR earlier, and this prediction score is thought to be a useful decision-making tool for the operation.

The limitations of this study are as follows. First, our study is a retrospective study and despite our efforts to control for confounding data and selection bias, it is not possible to control for all confounders. Second, as a single center study, our sample size is relatively small and susceptible to a type II error. Therefore we could not come to definite conclusions. However, the follow-up periods were more than 20 years, which was adequate for examining the effect of isolated AVR for chronic AR. 


\section{Conclusions}

The results of AVR for chronic AR were satisfactory in terms of early and late survival and no factor significantly affected the clinical results. However, for the preservation of postoperative normal LV systolic function, AVR for AR patients should be indicated before iESD reaches $26.7 \mathrm{~mm} / \mathrm{m}^{2}$. This result supports the new guidelines from the viewpoint of the LV reverse remodeling effect.

\section{Disclosure Statement}

All authors have no conflict of interest.

\section{References}

1) Tornos P, Sambola A, Permanyer-Miralda G, et al. Long-term outcome of surgically treated aortic regurgitation: influence of guideline adherence toward early surgery. J Am Coll Cardiol 2006; 47: 1012-7.

2) Henry WL, Bonow RO, Borer JS, et al. Observations on the optimum time for operative intervention for aortic regurgitation. I. Evaluation of the results of aortic valve replacement in symptomatic patients. Circulation 1980; 61: 471-83.

3) Taniguchi K, Sawa Y. Contemporary reviews by surgeon: timing of operation for chronic aortic regurgitation. Gen Thorac Cardiovasc Surg 2012; 60: 735-43.

4) Sambola A, Tornos P, Ferreira-Gonzalez I, et al. Prognostic value of preoperative indexed end-systolic left ventricle diameter in the outcome after surgery in patients with chronic aortic regurgitation. Am Heart J 2008; 155: 1114-20.

5) Brown ML, Schaff HV, Suri RM, et al. Indexed left ventricular dimensions best predict survival after aortic valve replacement in patients with aortic valve regurgitation. Ann Thorac Surg 2009; 87: 1170-5; discussion 1175-6.

6) Cho SH, Byun CS, Kim KW, et al. Preoperative indexed left ventricular dimensions to predict early recovery of left ventricular function after aortic valve replacement for chronic aortic regurgitation. Circ J 2010; 74: 2340-5.

7) Vahanian A, Alfieri O, Andreotti F, et al. Guidelines on the management of valvular heart disease (version 2012). The Joint Task Force on the Management of Valvular Heart Disease of the European Society of Cardiology (ESC) and the European Association for Cardio-Thoracic Surgery (EACTS) Eur Heart J; 2012; 33: 2451-96.

8) Nishimura RA, Otto CM, Bonow RO, et al. 2014 AHA/ACC guideline for the management of patients with valvular heart disease: executive summary: a report of the American College of Cardiology/American
Heart Association Task Force on Practice Guidelines. J Am Coll Cardiol 2014; 63: 2438-88.

9) Akins CW, Miller DC, Turina MI, et al. Guidelines for Reporting Mortality and Morbidity After Cardiac Valve Interventions. Ann Thorac Surg. 2008; 85: 1490-5.

10) Lang RM, Bierig M, Devereux RB, et al. Recommendations for chamber quantification: a report from the American Society of Echocardiography's Guidelines and Standards Committee and the Chamber Quantification Writing Group, developed in conjunction with the European Association of Echocardiography, a branch of the European Society of Cardiology. J Am Soc Echocardiogr 2005; 18: 1440-63.

11) Daimon M, Watanabe $H$, Abe $Y$, et al. Normal values of echocardiographic parameters in relation to age in a healthy Japanese population: the JAMP study. Circ J 2008; 72: 1859-66.

12) Sénéchal M, Bernier M, Dagenais F, et al. Usefulness of preoperative stroke volume as strong predictor of left ventricular remodeling and outcomes after aortic valve replacement in patients with severe pure aortic regurgitation. Am J Cardiol 2011; 108: 1008-13.

13) Enriquez-Sarano M, Tajik AJ. Clinical practice. Aortic regurgitation. N Engl J Med 2004; 351: 1539-46.

14) Chaliki HP, Mohty D, Avierinos JF, et al. Outcomes after aortic valve replacement in patients with severe aortic regurgitation and markedly reduced left ventricular function. Circulation 2002; 106: 2687-93.

15) Klodas E, Enriquez-Sarano M, Tajik AJ, et al. Aortic regurgitation complicated by extreme left ventricular dilation: long-term outcome after surgical correction. J Am Coll Cardiol 1996; 27: 670-7.

16) Iung $B$, Baron $G$, Butchart EG, et al. A prospective survey of patients with valvular heart disease in Europe: The Euro Heart Survey on Valvular Heart Disease. Eur Heart J 2003; 24: 1231-43.

17) Taniguchi K, Nakano S, Kawashima $Y$, et al. Left ventricular ejection performance, wall stress, and contractile state in aortic regurgitation before and after aortic valve replacement. Circulation 1990; 82: 798-807.

18) Bonow RO, Dodd JT, Maron BJ, et al. Long-term serial changes in left ventricular function and reversal of ventricular dilatation after valve replacement for chronic aortic regurgitation. Circulation 1988; 78: 1108-20.

19) Bhudia SK, McCarthy PM, Kumpati GS, et al. Improved outcomes after aortic valve surgery for chronic aortic regurgitation with severe left ventricular dysfunction. J Am Coll Cardiol 2007; 49: 1456-71.

20) Kamath AR, Varadarajan P, Turk R, et al. Survival in patients with severe aortic regurgitation and severe left ventricular dysfunction is improved by aortic valve replacement: results from a cohort of 166 patients with an ejection fraction $<$ or $=35 \%$. Circulation 2009; 120: S134-8. 
21) Bonow RO, Carabello BA, Chatterjee K, et al. Focused update incorporated into the ACC/AHA 2006 guidelines for the management of patients with valvular heart disease: a report of the American College of Cardiology/ American Heart Association Task Force on Practice Guidelines (writing committee to revise the 1998 guidelines for the management of patients with valvular heart disease): endorsed by the Society of Cardiovascular Anesthesiologists, Society for Cardiovascular Angiography and Interventions, and Society of Thoracic Surgeons. Circulation 2008; 118: e523-661.

22) Turk R, Varadarajan P, Kamath A, et al. Survival benefit of aortic valve replacement in older patients with asymptomatic chronic severe aortic regurgitation. Ann Thorac Surg 2010; 89: 731-7.

23) Kang HJ, Kim YJ, Sohn DW, et al. Prediction of postoperative left ventricular systolic function with Doppler-derived $\mathrm{dP} / \mathrm{dt}$ in patients with chronic aortic regurgitation. J Am Soc Echocardiogr 2003; 16: 1111-5.

24) Hirooka K, Yasumura Y, Tsujita Y, et al. Enhanced method for predicting left ventricular reverse remodeling after surgical repair of aortic regurgitation: application of ultrasonic tissue characterization. J Am Soc Echocardiogr 2002; 15: 695-701.

25) Sénéchal M, Lancellotti P, Garceau P, et al. Usefulness and limitation of dobutamine stress echocardiography to predict acute response to cardiac resynchronization therapy. Echocardiography 2010; 27: 50-7.

26) Tribouilloy C, Lévy F, Rusinaru D, et al. Outcome after aortic valve replacement for low-flow/low-gradient aortic stenosis without contractile reserve on dobutamine stress echocardiography. J Am Coll Cardiol 2009; 53: 1865-73.

27) Ling LH, Kistler PM, Ellims AH, et al. Diffuse ventricular fibrosis in atrial fibrillation: noninvasive evaluation and relationships with aging and systolic dysfunction. J Am Coll Cardiol 2012; 60: 2402-8.

28) Zhou X, Sheng Y, Yang R, et al. Nicotine promotes cardiomyocyte apoptosis via oxidative stress and altered apoptosis-related gene expression. Cardiology 2010; 115: 243-50.

29) Ibe W, Saraste A, Lindemann S, et al. Cardiomyocyte apoptosis is related to left ventricular dysfunction and remodeling in dilated cardiomyopathy, but is not affected by growth hormone treatment. Eur J Heart Fail 2007; 9: 160-7. 